\title{
Particle Pair Production in Cosmological General Relativity-Redux
}

\section{Firmin J. Oliveira}

East Asian Observatory/James Clerk Maxwell Telescope, Hilo, HI, USA

Email: firmjay@hotmail.com

How to cite this paper: Oliveira, F.J. (2020) Particle Pair Production in Cosmological General Relativity-Redux. Journal of High Energy Physics, Gravitation and Cosmology, 6, 34-42.

https://doi.org/10.4236/jhepgc.2020.61004

Received: October 18, 2019

Accepted: December 1, 2019

Published: December 4, 2019

Copyright $\odot 2020$ by author(s) and Scientific Research Publishing Inc. This work is licensed under the Creative Commons Attribution International License (CC BY 4.0).

http://creativecommons.org/licenses/by/4.0/

(c) (i) Open Access

\begin{abstract}
We model the universe on the interaction of two cosmic particles based on the Cosmological General Relativity (CGR) of Carmeli and obtain a theoretical value for the Hubble constant $h$ at zero distance and no gravity. CGR is a 5 -dimensional theory of time $t$, space $x, y, z$ and velocity $v$. A minimum cosmic acceleration $a_{0}=\mathrm{d} v / \mathrm{d} t=c / \tau$ results from a linearized version of CGR, where $c$ is the vacuum speed of light and $\tau$ is the Hubble-Carmeli time constant. The force due to the Carmeli acceleration $a_{0}$ counteracts the Newtonian gravitational force between the two particles. Each particle is unstable and disintegrates into baryons, leptons and radiation. By the uniform expansion of the black body radiation field, we obtain the expression $\tau=\sqrt{A /\left(T_{0}^{3}+\Omega_{\text {bphys }}(0.1 p c)^{-2} A\right)}$, where $A$ is a constant, $T_{0}$ is the temperature of the cosmic microwave background black body, $\Omega_{\text {bphys }}$ is the physical baryon density parameter and $p c \approx 3.086 \times 10^{18} \mathrm{~cm} \cdot \mathrm{pc}^{-1}$. Using standard values for $T_{0}$ and $\Omega_{\text {bphys }}$ we obtain a value $\tau=(4.15121 \pm 0.00206) \times 10^{17} \mathrm{~s}$, which gives a value for the Hubble constant at zero distance and no gravity of $h=1 / \tau=(74.33982 \pm 0.03694) \mathrm{km} \cdot \mathrm{s}^{-1} \cdot \mathrm{Mpc}^{-1}$. From the value for $\tau$, we get the age of the universe of $(13.15467 \pm 0.00653) \times 10^{9}$ years.
\end{abstract}

\section{Keywords}

Hubble Constant, Hubble-Carmeli Time Constant, Vacuum Density, Cosmic Particle

\section{Introduction}

The Cosmological General Relativity (CGR) of Carmeli is a 5-dimensional theory of time $t$, space $x, y, z$ and velocity $v$, which predicts the existence of a 
constant acceleration $a_{0}=c / \tau$ due to the expansion of the universe [1] [2], where $\mathrm{c}$ is the speed of light in vacuum and $\tau$ is the Hubble-Carmeli time constant and where $h=1 / \tau$ is the Hubble constant at zero distance and no gravity. In the application of Carmeli cosmology to galaxy rotation dynamics, Hartnett [3] found that the dividing line between which the galaxy rotation velocity was explained by Newtonian dynamics and where it could be explained by Carmeli dynamics, respectively, was where the galaxy acceleration transitioned from greater than a critical value of $(2 / 3) a_{0}$ to less than this acceleration.

We assume that the universe of mass and energy began with the formation of two massive cosmic particles. The pair of neutral cosmic particles materialized from the vacuum and the Carmeli acceleration formed the force which opposed the gravitational force between them. It is found that the particles were initially separated a distance $r_{0}=c \tau / 2$ and each had a mass $m=\tau c^{3} / 4 G$, where $G$ is Newton's gravitation constant. For the universe, the sum of the mass density $\rho$ of the particles (positive) and the mass density $\rho_{v a c}$ of the vacuum (negative) satisfies $\rho+\rho_{\text {vac }}=0$. We derive for the mass densities $\rho=-\rho_{\text {vac }}=\rho_{c}$, where $\rho_{c}=3 /\left(8 \pi G \tau^{2}\right)$ is the critical mass density. The particles are assumed to be the anti-particle of each other and inherently unstable. Upon the disintegration of each particle, the remnant of baryons, leptons and photons are hypothesized to form two sub-universes enclosed in a sphere of radius $R_{S}=c \tau$. The baryons, leptons and photons interact as they expand to fill the hemisphere of each subuniverse. When the radiation field photons reach a state of equilibrium with the ionized hydrogen atoms and electrons, the average photon energy $\varepsilon_{\gamma}=\alpha^{2} \mu c^{2} / 2$, where $\alpha$ is the fine structure constant and $\mu$ is the reduced electron mass in the hydrogen atom.

This paper is a summary of the main points of my earlier paper [4]. We will cover the essential ideas developed in detail there and while making a more rigorous derivation of our expression for the Hubble-Carmeli time constant.

\section{The Initial State of the Universe}

We take the natural position that the universe can be described by an equation of the form $U=0$, where $U$ represents a fundamental quantity such as the energy, mass density or force. Assuming that only gravitational and expansion reactions need be considered, ignoring the nuclear, electric and magnetic effects, we can state that the mass densities and forces due to gravity and expansion upon two cosmic particles each of the same mass are given by

$$
\begin{gathered}
\rho+\rho_{v a c}=0, \\
F\left(r_{0}\right)=\frac{G m^{2}}{r_{0}^{2}}-m a_{0}=0,
\end{gathered}
$$

where $\rho$ is the matter mass density of the universe, $\rho_{\text {vac }}$ is the vacuum mass density, $m$ is the mass of each of the cosmic particles, $F(r)$ is the sum of forces on each particle, $G$ is Newton's gravitation constant, $r_{0}$ is the separation of the two particles and $a_{0}=c / \tau$ is the Carmeli acceleration. 
Initially, the two particles are at rest relative to each other. It is assumed that each cosmic particle is the anti-particle of the other. For example, representing ordinary matter by $u$ and anti-matter by $\bar{u}$, if one particle is composed of $(x+y) u$ amount of ordinary matter and $x \bar{u}$ amount of antimatter such that $b_{1}=(x+y) u-x \bar{u}$, then the antiparticle is composed of $x u$ amount of ordinary matter and $(x+y) \bar{u}$ amount of antimatter, giving $b_{2}=x u-(x+y) \bar{u}$. Summing the compositions of both particles produces $b_{1}+b_{2}=y u-y \bar{u}=0$, conserving quantum numbers.

A further assumption we make is that both cosmic particles are enclosed in a finite spherical volume $V$ having a radius equal to the Schwarzschild radius $R_{S}$ of the particles given by

$$
R_{S}=\frac{2 G(2 m)}{c^{2}}
$$

Multiplying the density relations in (1) by the volume $V=(4 / 3) \pi R_{S}^{3}$ we get the equation for the total mass

$$
\frac{4}{3} \pi R_{S}^{3}\left(\rho+\rho_{v a c}\right)=2 m+\left(\frac{256 \pi G^{3} m^{3}}{3 c^{6}}\right) \rho_{v a c}=0,
$$

where we assume that the matter density $\rho=2 \mathrm{~m} / \mathrm{V}$. Multiply the force (2) by the differential distance and integrate to obtain the energy of particle \#2 at distance $r_{0}$ from particle \#1, which is given by

$$
E\left(r_{0}\right)=\int\left(\frac{G m^{2}}{r^{2}}-m a_{0}\right) \mathrm{d} r=-\frac{G m^{2}}{r_{0}}-m a_{0} r_{0}+m c^{2},
$$

where it is assumed that the integration constant is the particle rest energy. By symmetry, we know that the result is the same if we reversed the roles of the particles. By setting $E\left(r_{0}\right)=0$ in (5) and along with (2) where $F\left(r_{0}\right)=0$, we solve these quadratic equations simultaneously to obtain the mass $m$ and separation distance $r_{0}$, which are found to be given by

$$
m=\frac{\tau c^{3}}{4 G} .
$$

and

$$
r_{0}=\frac{c \tau}{2}
$$

Solving (3) for $R_{S}$ with the mass $m$ from (6) we obtain

$$
R_{S}=c \tau .
$$

Substituting for mass $m$ from (6) into (4), we obtain for the vacuum mass density

$$
\rho_{v a c}=\frac{-3}{8 \pi G \tau^{2}}
$$

and from (8) into (2), the matter mass density 


$$
\rho=-\rho_{\text {vac }}=\frac{3}{8 \pi G \tau^{2}}=\rho_{c},
$$

where $\rho_{c}$ is the critical mass density. Carmeli defined the effective mass density $\rho_{\text {eff }}$ in his theory in the form $\rho_{\text {eff }}=\rho-\rho_{c}$, where $\rho$ is the mass density, but the critical mass density did not have a physical aspect. In this work we attribute a negative mass density to the vacuum and define the effective mass density by $\rho_{\text {eff }}=\rho+\rho_{\text {vac }}$. Therefore, by (1) we imply that at the beginning,

$$
\rho_{\text {eff }}=0 \text {. }
$$

\section{Radiation Energy of the Universe in the Form of a Black Body}

The two cosmic particles, \#1 and \#2, are each of mass $\mathrm{m}$ and are assumed to be composed of subatomic particles of matter and anti-matter as we described above. It can be shown [4, Ref. 7] that each particle is enclosed in a volume $V_{m}$ which is half of the sphere volume $V$ of Schwarzschild radius $R_{S}$ given by (8) which encloses both particles, so that the volume of the sub-universe containing a single particle, let us say \#1, is given by

$$
V_{m}=\frac{1}{2} V=\frac{2 \pi}{3} c^{3} \tau^{3}
$$

Since each cosmic particle is composed of subatomic particles and anti-particles it is inherently unstable and will disintegrate, by particle anti-particle annihilations, yielding photons and a remnant of baryons and leptons. Assuming that a fraction $g$ of the particle mass $m$ ends up as baryons and leptons, so then a fraction $(1-g)$ of the mass $\mathrm{m}$ ends up as photons. Assume also that the baryons and leptons are at rest initially. By energy conservation, the total energy $E_{T}=m c^{2}=E_{B}+E_{\gamma}$, where the energy of the baryons and leptons is

$$
E_{B}=g m c^{2},
$$

and the energy of the photons is

$$
E_{\gamma}=(1-g) m c^{2} \text {. }
$$

The total energy density of radiation (both polarizations) in a black body at temperature $T[5]$ is given by

$$
u(T)=\frac{\pi^{2} k_{B}^{4} T^{4}}{15 c^{3} \hbar^{3}}
$$

where $k_{B}$ is Boltzmann's constant and $\hbar$ is the reduced Planck's constant. Multiplying the energy density (14) by the present sub-universe volume $V_{m}$ enclosing it we have the radiation total energy $E_{\gamma, 0}$ at the present time where the universe temperature is $T_{0}$

$$
E_{\gamma, 0}=u\left(T_{0}\right) V_{m}=\frac{2 \pi^{3} \tau^{3} k_{B}^{4} T_{0}^{4}}{45 \hbar^{3}} .
$$

At some point, the radiation field interaction with the baryons and leptons 
reaches a stable state such that the number of photons $N_{\gamma}(T)$ in the field remains fixed as temperature $T$ decreases due to the physical expansion of the radiation field. The average photon energy in the black body at temperature $T$ is expressed by

$$
\varepsilon_{\text {avg }}=\zeta k_{B} T,
$$

where $\zeta \approx 2.70$ is the black body mean energy coefficient. Dividing $E_{\gamma, 0}$ by $\varepsilon_{\text {avg }, 0}=\zeta k_{B} T_{0}$, which is the average CMB photon energy now, yields the number of photons at the present epoch of time in the sub-universe,

$$
N_{\gamma}\left(T_{0}\right)=\frac{E_{\gamma, 0}}{\varepsilon_{\text {avg }, 0}}=\frac{2 \pi^{3} \tau^{3} k_{B}^{3} T_{0}^{3}}{45 \zeta \hbar^{3}} .
$$

The temperature of the cosmic microwave background (CMB) in the universe at the present time is, $T_{0} \approx 2.73 \mathrm{~K}$ and using this temperature the number of photons in the radiation field, the CMB is given by (17), using a value of $\tau=4.28 \times 10^{17} \mathrm{~s}$

$$
N_{\gamma}\left(T_{0}\right) \approx 1.82 \times 10^{87} .
$$

Looking back to the time when the photon number first stabilized to a fixed value, which is assumed to be the same as the present number $N_{\gamma}\left(T_{0}\right)$, we make a first approximation of the average photon energy $\varepsilon_{\gamma}$ at that time by dividing the total sub-universe mass energy $m c^{2}$ by the number of photons, giving

$$
\varepsilon_{\gamma} \approx \frac{m c^{2}}{N_{\gamma}\left(T_{0}\right)} \approx 13.32 \mathrm{eV},
$$

which we realize is $98 \%$ of the ionization energy $13.6 \mathrm{eV}$ of the hydrogen atom. Because big bang nucleosynthesis ends with the radiation field interacting with the ionized hydrogen atoms, it is reasonable to hypothesize that the average photon energy equals the ionization energy,

$$
\varepsilon_{\gamma}=\frac{(1-g) m c^{2}}{N_{\gamma}\left(T_{0}\right)}=\frac{\alpha^{2} \mu c^{2}}{2}
$$

where the last expression on the right hand side is the hydrogen atom ionization energy, where $\alpha$ is the fine structure constant and $\mu$ is the reduced electron mass of the hydrogen atom. Equation (20) can be expanded using the mass $\mathrm{m}$ from (6) and $N_{\gamma}\left(T_{0}\right)$ from (17) which simplifies to give

$$
\varepsilon_{\gamma}=\frac{(1-g) 45 \zeta \hbar^{3} c^{5}}{8 \pi^{3} G \tau^{2} k_{B}^{3} T_{0}^{3}}=\frac{\alpha^{2} \mu c^{2}}{2} .
$$

As for the definition of the fractional parameter $g$ representing the baryons, we take a simpler form here than in [4], expressed by

$$
g=\frac{\Omega_{b p h y s}}{h_{c}^{2}}
$$

where $\Omega_{\text {bphys }}$ is the physical baryon density parameter and $h_{c}$ is the Hubble constant $h$ divided by 100, which are defined in the cgs system by 


$$
h=\frac{10 p c}{\tau},
$$

where $p c \approx 3.086 \times 10^{18} \mathrm{~cm} / \mathrm{pc}$ and

$$
h_{c}=\frac{h}{100} .
$$

Since $\varepsilon_{\gamma}=\zeta k_{B} T_{\gamma}$ from (16), the temperature of the black body radiation field during the hydrogen ionization epoch is given by

$$
T_{\gamma}=\frac{\varepsilon_{\gamma}}{\zeta k_{B}}=\frac{\alpha^{2} \mu c^{2}}{2 \zeta k_{B}} .
$$

Substituting values into (25) we get $T_{\gamma} \approx 5.84 \times 10^{4} \mathrm{~K}$.

\section{The Values of the Hubble-Carmeli Time Constant and the Hubble Constant}

Since it is difficult to measure the Hubble constant through astronomical methods we can invert Equation (21) to solve for $\tau$ in terms of $\Omega_{b p h y s}$ and $T_{0}$, parameters which may be more accurately determined. First, define the constant $A$ in the form

$$
A=\frac{45 \zeta \hbar^{3} c^{3}}{4 \pi^{3} G k_{B}^{3} \alpha^{2} \mu} .
$$

Then, from (22), (23) and (24) express $g$ in the form

$$
g=\frac{\Omega_{b p h y s} \tau^{2}}{(0.1 p c)^{2}} .
$$

Then, with (26) and (27) we invert (21) and solve it for $\tau$, yielding

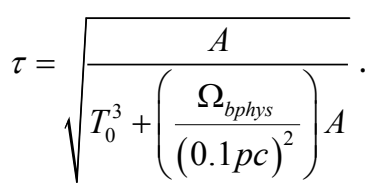

Substitute values into (28) for the parameters in $A$ from (26) and for the present day CMB temperature $T_{0}=2.72548 \pm 0.00057 \mathrm{~K}$ [6] and physical baryon density parameter $\Omega_{b p h y s}=0.02260 \pm 0.00034$ [7] to obtain a predicted value of

$$
\tau \approx(4.15121 \pm 0.00206) \times 10^{17} \mathrm{~s},
$$

which gives a value for $h$, the Hubble constant at zero distance and no gravity of

$$
h=(74.33982 \pm 0.03694) \mathrm{km} \cdot \mathrm{s}^{-1} \cdot \mathrm{Mpc}^{-1} \text {. }
$$

For the age of the universe, $t_{0}=\tau$ /sidereal year, we have

$$
t_{0}=(13.15467 \pm 0.00653) \times 10^{9} \mathrm{yr} .
$$

In [Oliveira 2012], we reported a similar value for $h$ as in (30), though the derivation in that paper was less rigorous than the formula (28) above. Recently, it was reported by [8] a value 
$H_{0}=74.03 \pm 1.42 \mathrm{~km} \cdot \mathrm{s}^{-1} \cdot \mathrm{Mpc}^{-1}$, also earlier by [9] a value $H_{0}=73.8 \pm 2.4 \mathrm{~km} \cdot \mathrm{s}^{-1} \cdot \mathrm{Mpc}^{-1}$, and by [10] a value $H_{0}=74.3 \pm 2.1 \mathrm{~km} \cdot \mathrm{s}^{-1} \cdot \mathrm{Mpc}^{-1}$ and also earlier by [11] a value $H_{0}=72 \pm 8 \mathrm{~km} \cdot \mathrm{s}^{-1} \cdot \mathrm{Mpc}^{-1}$, all of which fit well with our prediction.

\section{Baryon to Photon Number Density}

The photon number density $n_{\gamma, 0}$ at the present epoch is the energy density (14) divided by the average photon energy (16) at the CMB temperature $T_{0}$ given by

$$
n_{\gamma, 0}=\frac{\mu\left(T_{0}\right)}{\zeta k_{B} T_{0}}=\frac{\pi^{2} k_{B}^{3} T_{0}^{3}}{15 \zeta c^{3} \hbar^{3}} .
$$

Also, the baryon number density $n_{B, 0}$ at the present epoch can be obtained by dividing the baryon energy $E_{B}$ of (12) by the volume $V_{m}$ of (11) and the proton mass $m_{p}$, and using (22), (23) and (24) and simplifying we have,

$$
n_{B, 0}=\frac{E_{B}}{m_{p} V_{m}}=\frac{g \tau c^{3} / 4 G}{m_{p}(2 / 3) \pi c^{3} \tau^{3}}=\left(\frac{\Omega_{b p h y s}}{m_{p} 0.01 p c^{2}}\right)\left(\frac{3}{8 \pi G}\right) .
$$

From (32) and (33), we obtain the baryon to photon ratio at epoch

$$
\eta=\frac{n_{B, 0}}{n_{\gamma, 0}}=\left(\frac{\Omega_{b p h y s}}{m_{p} 0.01 p c^{2}}\right)\left(\frac{45 \zeta c^{3} \hbar^{3}}{8 G \pi^{3} k_{B}^{3} T_{0}^{3}}\right) .
$$

The expression (34) for $\eta$ can be put into a simpler form using (21) to substitute into the second term yielding,

$$
\eta=\frac{n_{B, 0}}{n_{\gamma, 0}}=\left(\frac{g}{m_{p} \tau^{2}}\right)\left(\frac{45 \zeta c^{3} \hbar^{3}}{8 G \pi^{3} k_{B}^{3} T_{0}^{3}}\right)=\left(\frac{g}{1-g}\right)\left(\frac{\alpha^{2} \mu}{2 m_{p}}\right),
$$

where $g$ is given by (27). Using the value of $\tau$ from (29) and $\Omega_{\text {bphys }}=0.02260 \pm 0.00034$ from [7] we get a theoretical value for the baryon to photon number ratio

$$
\eta_{10}=6.17955 \pm 0.11217,
$$

where $\eta_{10}=\eta \times 10^{10}$, which is within the range $\left(4.7 \leq \eta_{10} \leq 6.5\right)$ reported in a BBN study [12]. Since $g$ from (22) is a constant, we see that $\eta$ given by (35) is a constant as is expected, since the number of baryons and photons is assumed to be constant for this analysis.

\section{Conclusions}

A key result of this thesis is showing that the initial mass $m$ of the universe can be partitioned into the baryon mass $m_{B}=g m$ and the photon mass $m_{\gamma}=(1-g) m$ at the time of nucleosynthesis. This is based on equating the average photon energy $\varepsilon_{\gamma}$ to the Hydrogen ionization energy at the time of recombination (20), which is a very reasonable assumption. Within this context, there is not a requirement for any other masses, such as particles of dark matter or dark energy. There are several extended theories of gravity which elegantly address the issues of the shortcomings of General Relativity, such as dark matter, dark energy and 
the cosmological constant [13].

Bringing this paper back in a simpler form was important, due to the fact of the recent findings for the Hubble constant which appear to be approaching the value we obtained already in the original 2012 report. Also, we wanted to study the relationship between the cosmic model developed here and a new study of big bang nucleosynthesis for Carmeli cosmological theory which is available in draft form on ResearchGate [14].

\section{Conflicts of Interest}

The authors declare no conflicts of interest regarding the publication of this paper.

\section{References}

[1] Carmeli, M. (2000) Derivation of the Tully-Fisher Law: Doubts about the Necessity and Existence of Halo Dark Matter. International Journal of Theoretical Physics, 39, 1397-1404. https://doi.org/10.1023/A:1003642921142

[2] Carmeli, M. (2008) Relativity: Modern Large-Scale Spacetime Structure of the Cosmos. World Scientific, Singapore. https://doi.org/10.1142/6820

[3] Hartnett, J.G. (2006) Spiral Galaxy Rotation Curves Determined from Carmeli General Relativity. International Journal of Theoretical Physics, 45, 2118-2136. https://doi.org/10.1007/s10773-006-9178-0

[4] Oliveira, F.J. (2012) Particle Pair Production in Cosmological General Relativity. International Journal of Theoretical Physics, 51, 3993-4005. https://doi.org/10.1007/s10773-012-1291-7

[5] Reif, F. (1965) Fundamentals of Statistical and Thermal Physics. McGraw-Hill Book Co., New York, 373-378.

[6] Fixsen, D.J. (2009) The Temperature of the Cosmic Microwave Background. The Astrophysical Journal, 707, 916-920. https://doi.org/10.1088/0004-637X/707/2/916

[7] Cooke, R.J., Pettini, M., Nollett, K.M. and Jorgennson, R. (2016) The Primordial Deuterium Abundance of the Most Metal-Poor Damped Lyman- $\alpha$ System. The Astrophysical Journal, 830, 148. https://doi.org/10.3847/0004-637X/830/2/148

[8] Riess, A.G., Casertano, S., Yuan, W., Macri, L.M. and Scolnic, D. (2019) Large Magellanic Cloud Cepheid Standards Provide a 1\% Foundation for the Determination of the Hubble Constant and Stronger Evidence for Physics Beyond $\Lambda$ CDM. The Astrophysical Journal, 876, 85. https://doi.org/10.3847/1538-4357/ab1422

[9] Riess, A.G., Macri, L., Casertano, S., et al. (2011) A 3\% Solution: Determination of the Hubble Constant with the Hubble Space Telescope and Wide Field Camera 3. The Astrophysical Journal, 739, 119. https://doi.org/10.1088/0004-637X/730/2/119

[10] Freedman, W.L., Madore, B.F., Scowcroft, V., et al. (2012) Carnegie Hubble Program: A Mid-Infrared Calibration of the Hubble Constant. The Astrophysical Journal, 758, 24-33. https://doi.org/10.1088/0004-637X/758/1/24

[11] Freedman, W.L., Madore, B.F., Gibson, B.K., et al. (2001) Final Results From the Hubble Space Telescope Key Project to Measure the Hubble Constant. The Astrophysical Journal, 553, 47-72. https://iopscience.iop.org/article/10.1086/320638/pdf

[12] Fields, B. and Sarkar, S. (2006) Big-Bang Nucleosynthesis. Nuclear Physics A, 777, 208-225. https://doi.org/10.1016/j.nuclphysa.2004.10.033 
[13] Corda, C. (2009) Interferometric Detection of Gravitational Waves: the Definitive Test for General Relativity. International Journal of Modern Physics D, 18, 2275-2282. https://doi.org/10.1142/S0218271809015904

[14] Oliveira, F.J. (2019) Big Bang Nucleosynthesis in Carmeli Cosmology-Part I. 